\title{
PROPRIEDADES FUNCIONAIS DE AMIDO DE LEGUMINOSAS EM ASSOCIAÇÃO À HIDROCOLOIDES
}

\author{
MARÍLIA SBRAGIA DEL BEM* \\ LUIS FERNANDO POLESI** \\ SILENE BRUDER SILVEIRA SARMENTO***
}

\begin{abstract}
O objetivo deste estudo foi avaliar o efeito da adição de hidrocoloides (goma guar, xantana e carboximetilcelulose) nas propriedades físico-químicas e funcionais de amido de ervilha e de grão-de-bico. As gomas foram adicionadas nas concentrações de $0 ; 0,1 ; 0,5$ e $1 \%$ sobre o peso do amido. A adição dos hidrocoloides elevou a viscosidade da pasta do amido de ervilha, que normalmente é muito baixa, assim como a tendência à retrogradação de modo proporcional ao aumento na concentração das gomas. A adição dos hidrocoloides resultou na redução da transparência das pastas do amido de ervilha. A resposta à adição dos diferentes hidrocoloides sobre as propriedades de pasta do amido de grão-de-bico não foi uniforme como para amido de ervilha. Entretanto, todos os hidrocoloides adicionados reduziram a tendência à retrogradação das pastas. A transparência do amido de grão-de-bico tendeu a se intensificar pela adição de xantana e a se reduzir pela adição de guar. A associação amido-hidrocoloide pode ser utilizada para alterar as propriedades funcionais dos amidos, aumentando sua possibilidade de uso. Entretanto, o comportamento não é uniforme para os diferentes amidos, nem para os diferentes hidrocoloides.
\end{abstract}

PALAVRAS-CHAVE: AMIDO; GRÃO-DE-BICO; ERVILHA; HIDROCOLOIDES; PROPRIEDADES REOLÓGICAS.

* Graduanda, Curso de Ciências dos Alimentos, Escola Superior de Agricultura Luiz de Queiroz, Universidade de São Paulo (ESALQ/USP), Piracicaba, SP (e-mail: mariliasbragia@hotmail.com).

** Mestre em Ciência e Tecnologia de Alimentos, ESALQ/USP, Piracicaba, SP (e-mail: Ifpolesi@usp.br).

*** Doutora em Ciência dos Alimentos, ESALQ/USP, Piracicaba, SP (e-mail: sbssarme@esalq.usp.br). 


\section{INTRODUÇÃo}

Diversos setores industriais empregam o amido e derivados como ingredientes ou componentes básicos de produtos, ou ainda como aditivos (baixas quantidades) para melhorar sua fabricação, apresentação ou conservação (CEREDA et al., 2001).

O uso do amido, constituído pelos polissacarídeos amilose e amilopectina, é requerido pelas indústrias alimentícias de acordo com as diversas propriedades que apresentam, mas principalmente pelo seu efeito espessante e/ou gelificante. Seus grânulos podem ser gelatinizados mediante o aquecimento de suspensão amido-água. Durante esse fenômeno ocorre a ruptura das estruturas cristalinas dos grânulos, os quais absorvem água e intumescem irreversivelmente, adquirindo tamanho maior que o original. Após a gelatinização do amido, quando a temperatura é reduzida à temperatura ambiente, ocorre o rearranjo das moléculas por ligações de hidrogênio, fator que favorece a recristalização ou retrogradação do amido (PARKER e RING, 2001).

A retrogradação do amido deve ser minimizada para sua aplicação em alimentos, pois se trata da reconstrução de estrutura mais rígida devido às cadeias de amilose ficarem mais disponíveis para se rearranjarem durante o armazenamento do alimento, resultando em perda de água do sistema e endurecimento do produto final (MUNHOZ, WEBER e CHANG, 2004).

As propriedades reológicas do amido podem variar de acordo com diversos fatores como a fonte botânica, a concentração utilizada e a presença de outras substâncias no meio.

Os hidrocoloides têm sido estudados em produtos à base de amido visando a associação de propriedades. A incorporação de gomas em suspensões de amido pode melhorar a estabilidade, modificar as propriedades reológicas, facilitar o processamento, reduzir custos e controlar a umidade do produto final (CHAISAWANG e SUPHANTHARIKA, 2005). A literatura (MUNHOZ, WEBER e CHANG, 2004; CHAISAWANG e SUPHANTHARIKA, 2005; WANG, ZHENGYU e XIAOPING, 2007) mostra que a adição de hidrocoloides diversos a amidos de fontes mais convencionais, como o milho e a mandioca, modificou principalmente as propriedades de gelatinização e retrogradação dos amidos.

Os amidos de leguminosas apresentam elevados teores de amilose (30-65\%), cristalinidade do tipo $\mathrm{C}$ e fortes interações entre as cadeias de amilose (HOOVER e ZHOU, 2003). O amido de ervilha apresenta características interessantes, dentre as quais teores de amilose entre 33 e $88 \%$, variáveis conforme as cultivares (RATNAYAKE, HOOVER e WARKENTIN, 2002), enquanto o amido de grão-de-bico apresenta teores de amilose entre 29 e 34\% (SINGH, SANDHU e KAUR, 2004). Em função dos elevados teores de amilose, esses amidos mostram grande tendência a retrogradação.

O presente estudo visou avaliar o efeito da adição de hidrocoloides (goma guar, goma xantana e carboximetilcelulose) nas propriedades físico-químicas e funcionais de amido de ervilha e de grão-de-bico.

\section{MATERIAL E MÉTODOS}

\subsection{MATERIAL}

Como matérias-primas para extração do amido foram utilizadas a ervilha verde rugosa (Pisum sativum) cultivar Utrillo, adquirida junto à Companhia de Entrepostos e Armazéns Gerais de São Paulo (CEAGESP) e grão-de-bico (Cicer arietinum) cultivar Cícero, adquirido junto à EMBRAPA HORTALIÇAS, em Brasília/DF. Os hidrocoloides goma guar e carboximetilcelulose foram cedidos pela indústria PLURY QUÍMICA LTDA e a goma xantana pela CPKELCO.

\subsection{MÉTODOS}

\subsubsection{Extração do amido de ervilha}

Realizou-se a extração de amido de ervilha pelo método alcalino modificado de Davydova et al. (1995). Os grãos da ervilha foram debulhados, triturados em homogeneizador com água destilada na proporção 1:5 e passados sequencialmente pelas peneiras de 60 e de 325 mesh. O resíduo foi 
lavado com água destilada e o material peneirado teve o $\mathrm{pH}$ ajustado para 7,6 mediante $\mathrm{NaOH}$ 0,08 mol. $\mathrm{L}^{-1}$, visando promover a floculação das proteínas e a separação do amido. Centrifugouse o material a $1120 \times g$ por $15 \mathrm{~min}$, sendo o sobrenadante descartado. Centrifugou-se novamente o amido decantado em água destilada e passado pelas peneiras de 60 e 325 mesh. O amido em suspensão foi centrifugado por 5 vezes até estar totalmente limpo. O amido foi, então, coletado e desidratado em estufa com circulação de ar a $40^{\circ} \mathrm{C}$ por 12 horas. O amido seco foi moído em almofariz e passado pela peneira de 60 mesh.

\subsubsection{Extração do amido de grão-de-bico}

Extraiu-se o amido de grão-de-bico de acordo com Singh, Sandhu e Kaur (2004). Após maceração dos grãos em solução de bissulfito de sódio a $0,16 \%$ por $16 \mathrm{~h}$ a $50^{\circ} \mathrm{C}$, a água de maceração foi drenada e os grãos triturados em liquidificador com água destilada na proporção 1:1. O material foi peneirado (60 e 140 mesh) e centrifugado a $1120 \times g$ por 5 min. Descartou-se o sobrenadante, sendo o decantado suspendido em água destilada e centrifugado novamente por 3 vezes. $\mathrm{O}$ amido foi desidratado em estufa com circulação de ar a $40^{\circ} \mathrm{C}$ por 12 horas e o amido seco moído em almofariz e passado pela peneira de 60 mesh.

\subsubsection{Composição dos amidos extraídos}

Determinou-se o teor de umidade em analisador de umidade A\&D Company Limited, modelo MX- 50, utilizando $1 \mathrm{~g}$ da amostra na temperatura de $105^{\circ} \mathrm{C}$. Para avaliação do grau de pureza do amido foram analisados os teores de cinzas, proteínas e lipídeos das amostras segundo os métodos descritos pela AOAC (2006) e de fibra bruta conforme a AACC (1995). Determinou-se o teor de nitrogênio do amido pelo método micro-kjeldahl, usando o fator 6,25 para a conversão do teor de nitrogênio para proteína bruta. O teor de lipídeos das amostras foi avaliado em aparelho Soxleth, tendo como solvente o hexano. Determinou-se o conteúdo de cinza após calcinação das amostras em mufla por 2 horas a $550^{\circ} \mathrm{C}$. O teor de amilose aparente presente nos amidos foi determinado de acordo com a metodologia ISO6647 (ISO, 1987).

\subsubsection{Efeito da adição de diferentes hidrocoloides nas propriedades funcionais de pastas de amidos de leguminosas}

As pastas obtidas foram avaliadas a partir de suspensões aquosas contendo apenas o amido natural ou amido/hidrocoloide. Os hidrocoloides (guar, xantana e CMC) foram acrescidos à dispersão em concentrações de 0,1; 0,5 e 1\% sobre o peso do amido.

\subsubsection{Propriedades viscoamilográficas}

As propriedade viscoamilográficas foram avaliadas em aparelho Rapid ViscoAnalyser (RVAS4A, Newport Scientific, Warriewood, Austrália), utilizando-se $3 \mathrm{~g}$ de amostra (14\% de umidade) em $25 \mathrm{~g}$ de água. Utilizou-se a programação Standard Analysis 2 (NEWPORT SCIENTIFIC, 1998), sendo os parâmetros Temperatura de Pasta, Viscosidade Máxima, Tempo de Viscosidade Máxima, Quebra de Viscosidade, Viscosidade Final e Tendência à Retrogradação extraídos do viscoamilograma. As unidades utilizadas para a medida de viscosidade foram Rapid Visco Units (RVU).

\subsubsection{Sinérese}

Avaliou-se a sinérese segundo método baseado na diferença de peso do material antes e após centrifugação, de acordo com a metodologia de Funami et al. (2005), com adaptações. A suspensão $5 \%(\mathrm{p} / \mathrm{p})$ foi aquecida em banho de água fervente durante 15 minutos com agitação constante para o amido de grão-de-bico, usando-se autoclave a $120^{\circ} \mathrm{C}$ durante 30 minutos para o amido de ervilha. $\mathrm{O}$ uso da autoclave para esse amido torna-se necessário devido sua elevada temperatura de gelatinização (faixa de 56 a $90^{\circ} \mathrm{C}$ ) (POLESI, SARMENTO e ANJOS, 2011). Após a gelatinização, o sistema foi transferido para tubo de centrífuga de $15 \mathrm{~mL}$ tarado e armazenado em geladeira a $4^{\circ} \mathrm{C}$ durante 7 dias. Pesou-se o gel (PG) e centrifugou-se a $3000 \mathrm{~g}$ por 10 minutos. $\mathrm{O}$ 
sobrenadante foi vertido em béquer e levado à estufa a $105^{\circ} \mathrm{C}$ até peso constante. Determinou-se 0 peso do líquido sobrenadante (PLS) pela diferença entre o peso do béquer com o sobrenadante e o peso do béquer pós-estufa. Os dados foram representados pela média de triplicatas. Calculou-se a porcentagem de sinérese de acordo com a equação "a":

$$
\text { Sinérese }(\%)=\frac{P L S}{P G} \times 100
$$

\subsubsection{Transparência/Opacidade}

Avaliou-se a transparência/opacidade das pastas de acordo com Craig et al. (1989), com adaptações. Para o grão-de-bico preparou-se suspensão aquosa a $1 \%(\mathrm{p} / \mathrm{v})$ de amido ou de amido/ hidrocoloide em béquer, que foi aquecido em banho de água fervente por 30 minutos com agitação manual a cada 5 minutos. Para o amido de ervilha, as suspensões a 1\% ( $\mathrm{p} / \mathrm{v})$ foram aquecidas em béquer tampado utilizando-se autoclave a $120^{\circ} \mathrm{C}$ por 30 minutos. Posteriormente, a pasta foi resfriada em temperatura ambiente até $35^{\circ} \mathrm{C}$, sendo a transmitância lida em espectrofotômetro a $650 \mathrm{~nm}$. A pasta foi reservada e a transmitância avaliada novamente 24 horas após a primeira leitura. Utilizou-se água como branco para zerar o aparelho.

\subsubsection{Análise estatística}

Adotou-se delineamento experimental fatorial $3 \times 4$, tendo como fatores o tipo de hidrocoloide (CMC, guar e xantana) e porcentagens de hidrocoloide $(0 ; 0,1 ; 0,5 ; 1)$. Os resultados foram submetidos à análise de variância (ANOVA) e ao Teste de Tukey $(p<0,05)$ para comparação de médias, utilizando-se o sistema estatístico SAS versão 9.1 (SAS, 2002/2003).

\section{RESULTADOS E DISCUSSÃO}

\subsection{COMPOSIÇÃO FÍSICO-QUÍMICA DOS AMIDOS}

O teor de umidade dos amidos de ervilha e de grão-de-bico (Tabela 1) ficou abaixo de $14 \%$ e, portanto, mostrou-se adequado para sua conservação até o momento da realização das análises.

TABELA 1 - COMPOSIÇÃO FÍSICO-QUÍMICA DOS AMIDOS

\begin{tabular}{ccccccc}
\hline Amido & $\begin{array}{c}\text { Umidade } \\
(\%)\end{array}$ & $\begin{array}{c}\text { Cinzas } \\
\left(\% \mathrm{bs}^{*}\right)\end{array}$ & $\begin{array}{c}\text { Proteínas } \\
(\% \mathrm{bs})\end{array}$ & $\begin{array}{c}\text { Lipídeos } \\
(\% \mathrm{bs})\end{array}$ & $\begin{array}{c}\text { Fibra } \\
(\% \mathrm{bs})\end{array}$ & $\begin{array}{c}\text { Amilose } \\
(\% \mathrm{bs})\end{array}$ \\
\hline Ervilha & 6,0 & 0,05 & 0,56 & 0,14 & 0,26 & 51,7 \\
Grão-de-bico & 12,4 & 0,10 & 0,20 & 0,15 & 0,24 & 30,2 \\
\hline
\end{tabular}

* base seca

O teor de cinzas no amido de ervilha coincidiu com os obtidos por Han e Tyler (2003), Hoover e Ratnayake (2002) e Ratnayake et al. (2001) de 0,03-0,14\%. No caso do grão-de-bico, o teor de cinza foi superior a 0,03-0,08\% encontrado por Singh, Sandhu e Kaur (2004).

O teor de proteínas no amido de ervilha mostrou-se superior ao obtido por Han e Tyler (2003), que variou de $0,22-0,27 \%$. O amido de grão-de-bico apresentou teor de proteína inferior à faixa de 0,69-089\%, encontrada na literatura (SINGH, SANDHU e KAUR, 2004).

O teor de lipídeos do amido de grão-de-bico citado na literatura varia de 0,04-0,52\% (SINGH, SANDHU e KAUR, 2004). No caso do amido de ervilha, os resultados ficaram acima dos valores de 0,03 a 0,05\%, citados na literatura (HAN e TYLER, 2003; RATNAYAKE et al., 2001).

Os baixos teores de cinzas, proteínas, lipídeos e fibra bruta tanto do amido de ervilha quanto do amido de grão-de-bico mostram a pureza dos amidos extraídos, assim como a eficiência do processo de extração. A somatória das substâncias acompanhantes (proteínas, cinzas, lipídeos 
e fibra) foi de 1,01\% para amido de ervilha e 0,69\% para amido de grão-de-bico. Esses valores são considerados baixos para interferir nas propriedades físico-químicas a serem analisadas subsequentemente.

O teor de amilose para o amido de grão-de-bico $(30,1 \%)$ enquadrou-se na faixa citada na literatura, entre 27,2 a 34,3\% (HUANG et al., 2007; SINGH, SANDHU e KAUR, 2004). O amido de ervilha apresentou $51,7 \%$ de amilose, acima do encontrado por Ratnayake et al. (2001), entre 42,9 a $43,7 \%$, em quatro diferentes cultivares de ervilha.

As diferenças nos valores dos componentes do amido com relação à literatura podem decorrer de diferentes variedades utilizadas, bem como de diversas metodologias de determinação.

\subsection{EFEITO DA ADIÇÃO DE HIDROCOLOIDES NAS PROPRIEDADES DE PASTA DOS AMIDOS}

A viscosidade das pastas de amido de ervilha, de modo geral, apresentaram valores muito baixos (Tabela 2). Para o amido natural de ervilha, a baixa viscosidade permaneceu durante toda a programação tempo/temperatura do viscógrafo (Figura 1). De forma semelhante, Polesi, Sarmento e Anjos (2011) encontraram em amido de ervilha natural da mesma cultivar valores de 1,$9 ; 0,5 ; 2,2$ e $0,8 \mathrm{RVU}$ para viscosidade máxima, quebra, final e tendência à retrogradação, respectivamente. Os autores atribuíram a viscosidade reduzida da pasta ao elevado teor de amilose dessa cultivar de ervilha, que dificulta a expansão dos grânulos durante o processo de gelatinização e, consequentemente, prejudica o aumento da viscosidade da pasta.

\section{TABELA 2 - PROPRIEDADES DE PASTA DOS AMIDOS DE ERVILHA}

\begin{tabular}{|c|c|c|c|c|c|c|}
\hline & \multirow{2}{*}{$\begin{array}{c}\text { Tempera- } \\
\text { tura de } \\
\text { pasta }\left({ }^{\circ} \mathrm{C}\right)\end{array}$} & \multirow{2}{*}{$\begin{array}{l}\text { Tempo de } \\
\text { pico (min) }\end{array}$} & \multicolumn{4}{|c|}{ Viscosidade (RVU) } \\
\hline & & & Máxima & Quebra & Final & $\begin{array}{c}\text { Tendência à } \\
\text { Retrogradacão }\end{array}$ \\
\hline $0 \%$ & $\ldots$ & $10,5 \pm 2,3$ & $1,9 \pm 0,2$ & $0,8 \pm 0$ & $1,8 \pm 0,1$ & $0,7 \pm 0,3$ \\
\hline $1 \% X$ & $\ldots$ & $11,3 \pm 0,9$ & $7,6 \pm 0,3$ & $1,1 \pm 0,3$ & $7,9 \pm 0,1$ & $1,4 \pm 0,1$ \\
\hline $\begin{array}{c}0,5 \% \\
\times\end{array}$ & $\ldots$ & $11,4 \pm 1,0$ & $4,9 \pm 0$ & $0,9 \pm 0,1$ & $4,9 \pm 0,1$ & $0,9 \pm 0,1$ \\
\hline $\begin{array}{c}\hat{\lambda} \\
\mathbf{X} \\
\mathrm{X}\end{array}$ & $\ldots$ & $11,0 \pm 1,6$ & $2,7 \pm 0$ & $1,2 \pm 0,1$ & $2,1 \pm 0,1$ & $0,6 \pm 0$ \\
\hline $1 \% \mathrm{G}$ & $\ldots$ & $12,0 \pm 0,1$ & $3,0 \pm 0,1$ & $0,6 \pm 0,1$ & $4,6 \pm 0,1$ & $2,2 \pm 0,1$ \\
\hline $\begin{array}{c}0,5 \% \\
G\end{array}$ & $\ldots$ & $12,4 \pm 0,7$ & $2,5 \pm 0,2$ & $0,8 \pm 0,1$ & $3,1 \pm 0,1$ & $1,4 \pm 0,2$ \\
\hline $\begin{array}{c}0,1 \% \\
G\end{array}$ & $\cdots$ & $10,8 \pm 1,3$ & $2,0 \pm 0,1$ & $0,9 \pm 0,2$ & $2,1 \pm 0,0$ & $1,0 \pm 0,3$ \\
\hline $\begin{array}{c}1 \% \\
\mathrm{CMC}\end{array}$ & $\cdots$ & $10,8 \pm 0,5$ & $4,6 \pm 0,1$ & $1,2 \pm 0,3$ & $5,3 \pm 0,3$ & $1,9 \pm 0,1$ \\
\hline $\begin{array}{l}0,5 \% \\
\text { CMC }\end{array}$ & $\ldots$ & $10,8 \pm 0,7$ & $3,5 \pm 0$ & $1,1 \pm 0,1$ & $3,7 \pm 0,1$ & $1,3 \pm 0,1$ \\
\hline $\begin{array}{l}0,1 \% \\
\text { CMC }\end{array}$ & $\cdots$ & $12,7 \pm 0,5$ & $2,2 \pm 0,2$ & $1,2 \pm 0,3$ & $2,2 \pm 0,7$ & $1,2 \pm 0,8$ \\
\hline
\end{tabular}

Notas: X - goma xantana; G - goma guar; CMC - goma CMC; $1 \%$ - adição de $1 \%$ de goma; $0,5 \%$ - adição de 0,5\% de goma; $0,1 \%$ - adição de $0,1 \%$ de goma.

Sinais convencionais utilizados: ... Dado numérico não disponível.

A análise dos perfis de RVA mostrou que a viscosidade do amido de ervilha natural foi sempre inferior às viscosidades do amido adicionado de qualquer uma das gomas. $\mathrm{O}$ aumento de viscosidade observado mostrou-se diretamente proporcional à concentração das gomas. A viscosidade final do amido de ervilha natural (1,75 RVU), por exemplo, aumentou com a adição de $0,1 \%, 0,5 \%$ e $1 \%$ de goma xantana apresentando, respectivamente, 2,1 RVU, 4,9 RVU e 7,9 RVU. Outros autores, como Korus etal. (2004) observaram o mesmo comportamento ao estudarem o efeito das gomas xantana e guar sobre o amido de triticale, cujas formulações apresentaram valores mais elevados de viscosidade de pasta e de força de cisalhamento que o amido nativo. A tendência à retrogradação desse amido também se elevou com a adição dos três tipos de gomas de 
modo gradativo, conforme o aumento da concentração de goma. Essas considerações, entretanto, envolvem valores muito baixos de retrogradação (entre 0,6 e 2,2\%).

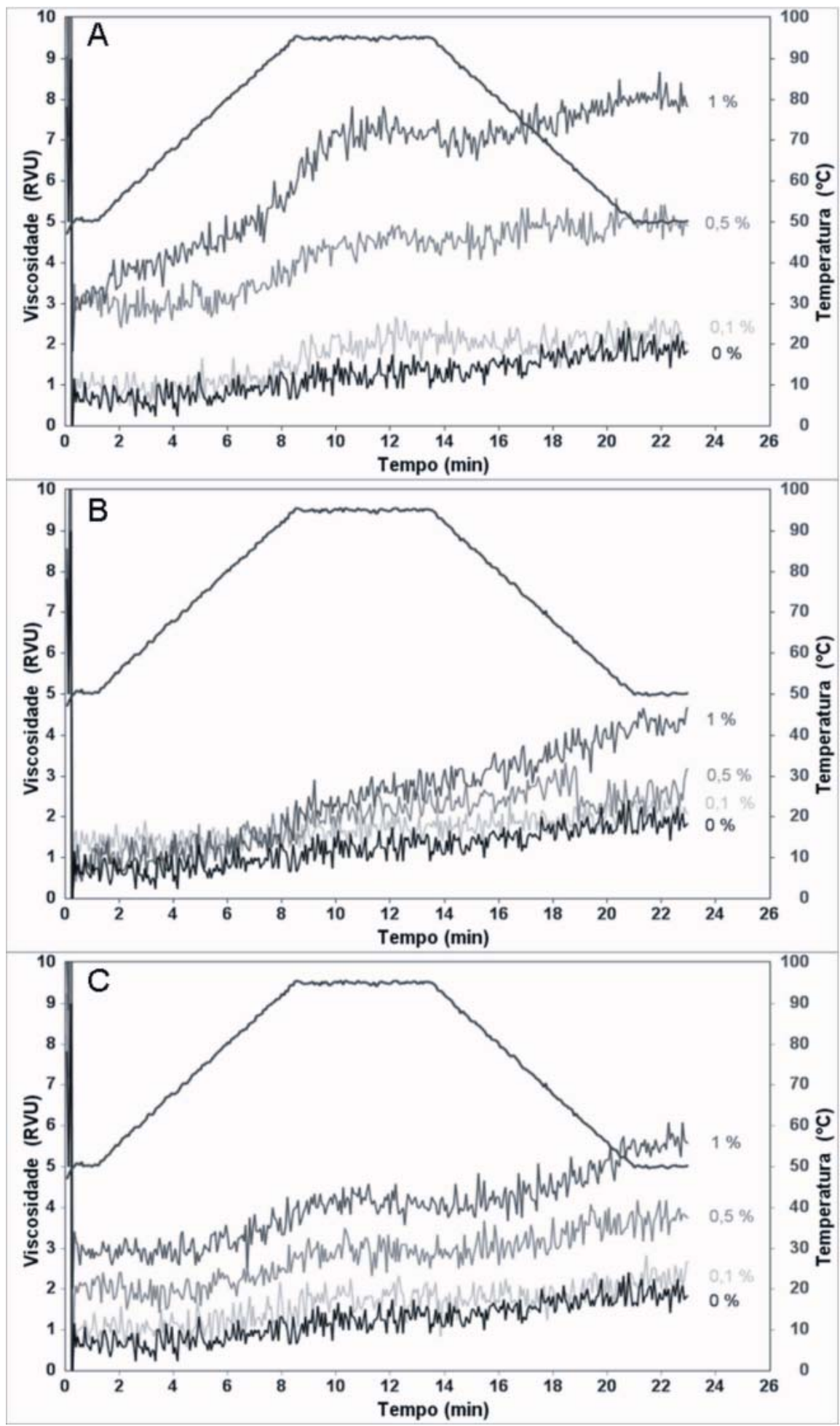

FIGURA 1 - PERFIS DE VISCOSIDADE DO AMIDO DE ERVILHA OBTIDOS NO RVA

$\mathrm{A}=$ amido + goma xantana; $\mathrm{B}=$ amido + goma guar $; \mathrm{C}=$ amido $+\mathrm{CMC}$.

O aumento da retrogradação pela adição de goma guar foi também observado por Chaisawang e Suphantharika (2006) para amido de mandioca nativo. Outros autores encontraram 
redução de retrogradação com a adição de gomas xantana e guar em amidos de milho, arroz e mandioca (WEBER et al., 2009; PONGSAWATMANIT e SRIJUNTHONGSIRI, 2008; SONG et al., 2006).

A viscosidade do amido de grão-de-bico (Tabela 3) foi mais elevada que a de amido de ervilha. O amido natural apresentou pico de viscosidade e temperatura de pasta bem definidos, certa tendência à quebra devido ao cisalhamento a quente e forte tendência à retrogradação (Figura 2), com viscosidade final do gel de $488 \mathrm{RVU}$.

TABELA 3 - PROPRIEDADES DE PASTA DOS AMIDOS DE GRÃO-DE-BICO

\begin{tabular}{ccccccc}
\hline & \multirow{2}{*}{$\begin{array}{c}\text { Temperatura } \\
\text { de Pasta }\left({ }^{\circ} \mathrm{C}\right)\end{array}$} & $\begin{array}{c}\text { Tempo de } \\
\text { Pico }(\mathrm{min})\end{array}$ & Máxima & Quebra & Final & $\begin{array}{c}\text { Tendência à } \\
\text { Retrogradação }\end{array}$ \\
\cline { 5 - 7 } & & & & & & \\
& $69,4 \pm 0,5$ & $6,5 \pm 0,1$ & $294,0 \pm 0,1$ & $114,8 \pm 5,1$ & $487,6 \pm 2,1$ & $308,5 \pm 7,1$ \\
\hline $1 \% \mathrm{X}$ & $\ldots$ & $9,4 \pm 0,3$ & $98,7 \pm 3,4$ & $17,0 \pm 2,1$ & $143,1 \pm 6,9$ & $61,3 \pm 5,7$ \\
\hline $0,5 \% \mathrm{X}$ & $\ldots$ & $6,9 \pm 0,1$ & $181,8 \pm 11,8$ & $70,3 \pm 2,4$ & $295,4 \pm 23,2$ & $183,9 \pm 13,7$ \\
\hline $0,1 \% \mathrm{X}$ & $71,0 \pm 0,7$ & $6,8 \pm 0$ & $269,1 \pm 6,7$ & $112,3 \pm 0,5$ & $433,0 \pm 9,1$ & $276,2 \pm 2,8$ \\
\hline $1 \% \mathrm{G}$ & $70,2 \pm 0,3$ & $6,9 \pm 0$ & $346,5 \pm 0,4$ & $108,7 \pm 6,1$ & $525,7 \pm 6,9$ & $287,9 \pm 0,4$ \\
\hline $0,5 \% \mathrm{G}$ & $70,6 \pm 0,9$ & $6,8 \pm 0$ & $313,0 \pm 2,4$ & $111,3 \pm 1,5$ & $494,8 \pm 0,1$ & $293,1 \pm 0,1$ \\
\hline $0,1 \% \mathrm{G}$ & $69,6 \pm 0,1$ & $6,3 \pm 0,1$ & $288,2 \pm 1,3$ & $112,8 \pm 4,6$ & $472,5 \pm 2,2$ & $297,0 \pm 3,7$ \\
\hline $1 \% \mathrm{CMC}$ & $\ldots$ & $9,1 \pm 0,1$ & $189,5 \pm 18,6$ & $34,3 \pm 5,4$ & $393,5 \pm 33,1$ & $238,3 \pm 19,9$ \\
\hline $0,5 \% \mathrm{CMC}$ & $\ldots$ & $7,4 \pm 0,1$ & $181,0 \pm 1,1$ & $35,5 \pm 3,6$ & $351,9 \pm 0,7$ & $206,5 \pm 1,7$ \\
\hline $0,1 \% \mathrm{CMC}$ & $70,1 \pm 0,1$ & $6,6 \pm 0,2$ & $252,0 \pm 0,5$ & $101,2 \pm 0,5$ & $400,7 \pm 2,4$ & $249,9 \pm 2,4$ \\
\hline
\end{tabular}

Notas: X - goma xantana; G - goma guar; CMC - goma CMC; $1 \%$ - adição de 1\% de goma; 0,5\% - adição de 0,5\% de goma; $0,1 \%$ - adição de $0,1 \%$ de goma.

Sinais convencionais utilizados: ... Dado numérico não disponível.

Para os amidos adicionados de 0,5 e 1\% de goma xantana e de 0,5 e $1 \%$ de CMC não foi verificada temperatura de pasta, sendo que para o restante das formulações a temperatura de pasta permaneceu em torno de $70^{\circ} \mathrm{C}$. Singh, Sandhu e Kaur (2004) encontraram valores entre 75,1$77,1^{\circ} \mathrm{C}$ para suspensões de amido (6\%) de grão-de-bico de diferentes cultivares.

A modificação da viscosidade das pastas de amido de grão-de-bico adicionadas de goma xantana e de CMC foi inversamente proporcional ao aumento da concentração de goma. Esse comportamento mostrou-se oposto ao que ocorreu com a adição de goma guar e diferiu do efeito dessas gomas no amido de ervilha.

As modificações causadas pelas gomas nas propriedades de pasta do amido devem-se à associação da goma com o amido intumescido ou com a amilose solúvel e as frações de amilopectina de massa molecular baixa, ou pela competição da goma com o amido pela água livre no sistema. As gomas modificam a reologia das pastas de amido e sua viscosidade contribui para a viscosidade do sistema e também pelo efeito na expansão dos grânulos durante e após a gelatinização (WEBER et al., 2009). Isso pode explicar o comportamento diferente entre as fontes de amido, ou seja, como a viscosidade do amido de ervilha é muito baixa, a viscosidade que a goma imprime ao sistema é detectada. No caso do amido de grão-de-bico com viscosidade elevada, o que predomina são as interações das gomas com a expansão dos grânulos.

Com relação à tendência a retrogradação, todas as gomas adicionadas ao amido de grãode-bico reduziram esse parâmetro. Além disso, a redução tendeu a ser diretamente proporcional ao aumento da concentração de goma adicionada, exceto a 0,5\% de CMC. Essa redução da retrogradação está relacionada tanto à supressão do intumescimento dos grânulos, que dificulta a gelatinização, quanto à associação das gomas com as moléculas de amilose que atrapalha a retrogradação. 

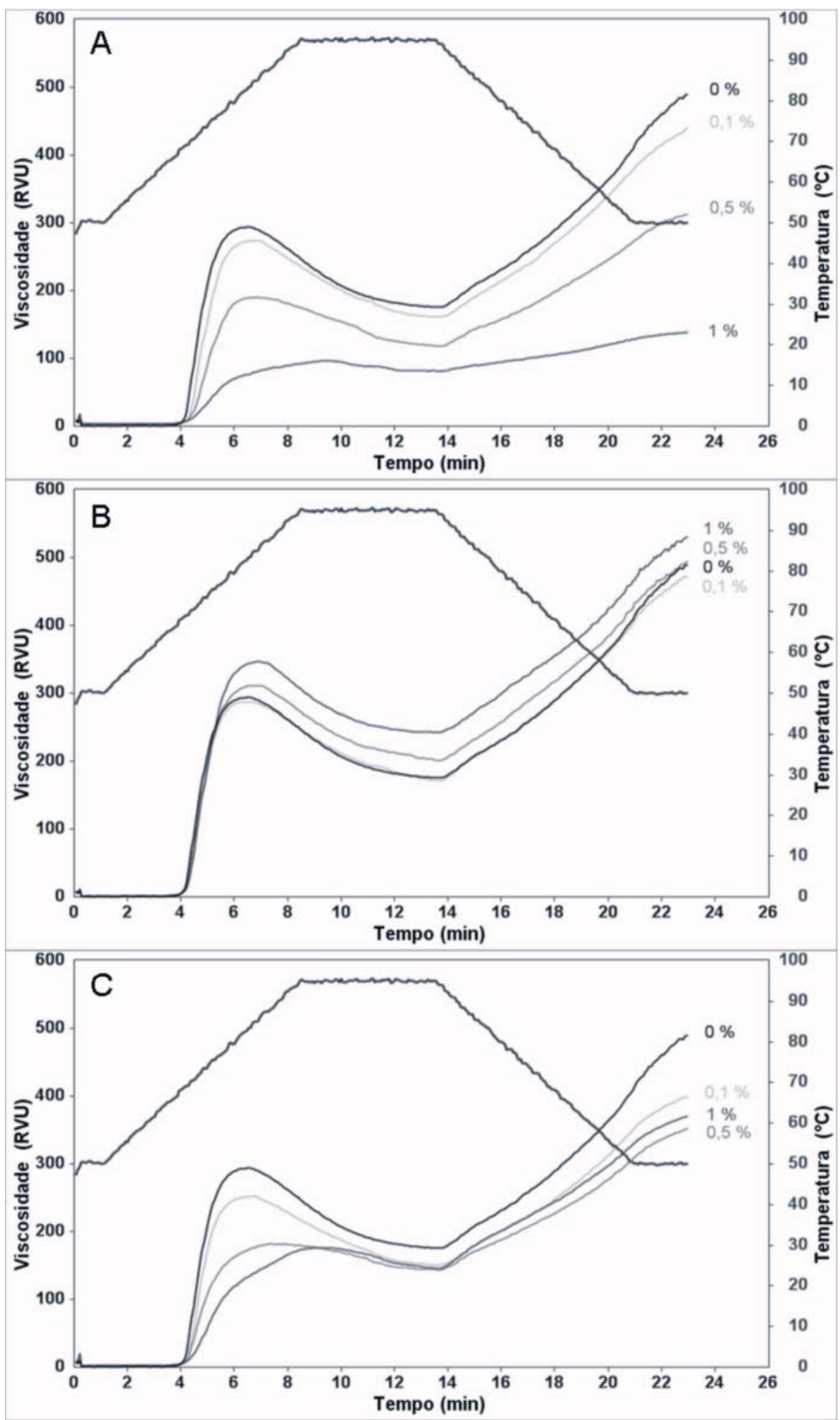

FIGURA 2 - PERFIS DE VISCOSIDADE DO AMIDO DE GRÃO-DE-BICO OBTIDOS NO RVA

$\mathrm{A}=$ amido + goma xantana $; \mathrm{B}=$ amido + goma guar $; \mathrm{C}=$ amido $+\mathrm{CMC}$.

\subsection{EFEITO DA ADIÇÃO DE HIDROCOLOIDES SOBRE A SINÉRESE DOS AMIDOS}

A sinérese dos géis de amido de ervilha (Tabela 4) associadas ou não aos hidrocoloides estudados tendeu a não diferir significativamente entre si em cada concentração avaliada, com exceção para $0,5 \%$ de goma xantana. 


\section{TABELA 4 - SINÉRESE (\%) DAS PASTAS DE AMIDO DE ERVILHA ASSOCIADA À HIDROCOLOIDES}

\begin{tabular}{cccc}
\hline$\%$ de goma & Xantana & Guar & CMC \\
\hline $1 \%$ & $25,7 \pm 10,7^{\mathrm{Aa}}$ & $23,5 \pm 3,4^{\mathrm{Aa}}$ & $28,1 \pm 6,5^{\mathrm{Aa}}$ \\
$0,5 \%$ & $5,5 \pm 0,7^{\mathrm{Bb}}$ & $15,8 \pm 11,6^{\mathrm{Aab}}$ & $28,7 \pm 4,5^{\mathrm{Aa}}$ \\
$0,1 \%$ & $9,7 \pm 1,8^{\mathrm{Ab}}$ & $14,9 \pm 8,8^{\mathrm{Aab}}$ & $4,8 \pm 0,5^{\mathrm{Ab}}$ \\
$0 \%$ & $4,6 \pm 0,3^{\mathrm{Ab}}$ & $4,6 \pm 0,3^{\mathrm{Ab}}$ & $4,6 \pm 0,3^{\mathrm{Ab}}$ \\
\hline
\end{tabular}

Médias seguidas pela mesma letra maiúscula na linha e mesma letra minúscula na coluna não diferem entre si pelo Teste de Tukey ao nível de 5\% de probabilidade.

A adição dos três hidrocoloides afetou consideravelmente a sinérese do amido de ervilha, sendo observada tendência geral a intensificar esse comportamento conforme a concentração utilizada. Esses resultados concordam com os observados para tendência a retrogradação desse amido obtida no RVA, pois quanto maior a re-associação das macromoléculas maior será a expulsão de água pelo gel.

A adição de CMC modificou a exsudação de água a partir da concentração de 0,5\%. O efeito da adição dessa goma parece variar de acordo com a fonte de amido. No estudo de Sudhakar, Singhal e Kulkarni (1992) foi observado comportamento oposto, cujos géis de amido de milho adicionados de CMC apresentaram estabilidade maior ao congelamento/descongelamento, ou seja, sinérese menor se comparados ao gel controle (sem goma).

LEE et al. (2002) estudaram o comportamento de géis de amido de batata-doce submetidos a cinco ciclos de congelamento e descongelamento adicionados de várias gomas nas concentrações de 0,3 e $0,6 \%$. Observaram que as gomas curdlana, gelana e carragena promoveram o aumento da sinérese do gel, ao contrário das demais gomas. Atribuíram esse fato à tendência de associação intermolecular das gomas que pode causar sinérese relativamente alta e baixa estabilidade do gel de amido. Além disso, verificaram que a goma xantana em excesso $(0,6 \%)$, devido à tendência de interações intermoleculares, pode desestabilizar e endurecer o gel.

A sinérese do gel de amido de grão-de-bico (Tabela 5) foi, em geral, superior à do gel de amido de ervilha. Isso ocorre porque em amidos com teor de amilose elevado há maior número de moléculas ramificadas que inibem a habilidade da amilose para formar duplas hélices longas, limitando o desenvolvimento de agregados estáveis (KLUCINEC e THOMPSON, 1999). Além disso, segundo Polesi, Sarmento e Anjos, (2011), o amido de ervilha com alto teor de amilose, mesmo com o processo de autoclavagem, não perde totalmente sua estrutura granular devido à baixa expansão dos grânulos, portanto, não libera as cadeias de amilose na sua totalidade. Weber, Queiroz e Chang (2008) observaram comportamento semelhante entre amido de milho normal ( $27 \%$ de amilose) com $75 \%$ de sinérese e amido de milho de alto teor de amilose $(71 \%$ de amilose) com $63 \%$ de sinérese.

\section{TABELA 5 - SINÉRESE (\%) DAS PASTAS DE AMIDO DE GRÃO-DE-BICO ASSOCIADA À HIDROCOLOIDES}

\begin{tabular}{cccc}
\hline \% de goma & Xantana & Guar & CMC \\
\hline $1 \%$ & $14,3 \pm 0,3^{\mathrm{Cc}}$ & $21,3 \pm 2,4^{\mathrm{Bc}}$ & $30,1 \pm 0,1^{\mathrm{Ac}}$ \\
$0,5 \%$ & $17,6 \pm 0,7^{\mathrm{Cb}}$ & $34,8 \pm 1,5^{\mathrm{Ab}}$ & $29,5 \pm 1,7^{\mathrm{Bc}}$ \\
$0,1 \%$ & $33,2 \pm 0,1^{\mathrm{Ba}}$ & $38,6 \pm 1,0^{\mathrm{Aa}}$ & $40,5 \pm 2,1^{\mathrm{Aa}}$ \\
$0 \%$ & $33,9 \pm 1,1^{\mathrm{Aa}}$ & $33,9 \pm 1,1^{\mathrm{Ab}}$ & $33,9 \pm 1,1^{\mathrm{Ab}}$ \\
\hline
\end{tabular}

Médias seguidas pela mesma letra maiúscula na linha e mesma letra minúscula na coluna não diferem entre si pelo Teste de Tukey ao nível de $5 \%$ de probabilidade.

A sinérese dos géis de amido de grão-de-bico diferiu conforme os hidrocoloides utilizados 
e cada concentração, comportamento diferente do observado para os géis de amido de ervilha. $\mathrm{Na}$ concentração de $1 \%$ de goma ocorreu a maior redução da sinérese do gel de amido e, dentre as três gomas, a xantana apresentou a maior redução nessa concentração.

As maiores concentrações (0,5 e 1\%) de goma nos géis de amido de grão-de-bico promoveram redução da sinérese, comportamento oposto ao observado para o amido de ervilha. O percentual de redução da sinérese verificado para adição de $1 \%$ de goma foi maior para a goma xantana (58\%) e menor para a CMC (11\%). Weber, Queiroz e Chang (2008) observaram redução de $11 \%$ na sinérese em gel de amido de milho normal com a adição de 1\% de goma xantana.

A xantana diminui os efeitos adversos do congelamento, impedindo a formação de estrutura esponjosa, resultante da retrogradação da amilose. As moléculas de amilose que foram lixiviadas dos grânulos no processo de gelatinização ficam expostas e podem estabelecer novas ligações moleculares com a goma xantana. Esse fato reduz a possibilidade de associações amiloseamilose, que são as principais ligações envolvidas no processo de retrogradação, pois quando essas moléculas se re-associam ocorre a expulsão da água intramolecular do gel dando origem à sinérese (LEE et al., 2002).

A goma guar e a CMC em doses baixas $(0,1 \%)$ apresentaram maior exsudação de água que o gel de amido de grão-de-bico controle.

\subsection{EFEITO DA ADIÇÃO DE HIDROCOLOIDES SOBRE A TRANSPARÊNCIA/OPACIDADE DAS PASTAS E GÉIS DE AMIDO}

A transparência/opacidade constitui importante atributo de qualidade pela aparência que conferem aos géis. A adição das gomas xantana, guar e $\mathrm{CMC}$ ao amido de ervilha tendeu a reduzir a transparência das pastas (Tabela 6). A goma guar a 0,1\% não apresentou valor de transmitância significativamente diferente da pasta de amido controle.

\section{TABELA 6 - TRANSMITÂNCIA (T\% a 650 nm) DAS PASTAS (0 hora) E GÉIS (24 horas) DE AMIDO DE ERVILHA}

\begin{tabular}{|c|c|c|c|}
\hline$\%$ de goma & Xantana & Guar & $\mathrm{CMC}$ \\
\hline \multicolumn{4}{|c|}{0 hora } \\
\hline $1 \%$ & $4,4 \pm 0,1 \mathrm{Bc}$ & $4,8 \pm 0,1^{\mathrm{Ab}}$ & $4,8 \pm 0,1 \mathrm{Ab}$ \\
\hline $0,5 \%$ & $4,7 \pm 0,2^{\mathrm{Ab}}$ & $4,1 \pm 0,1^{\mathrm{Bc}}$ & $4,8 \pm 0,1^{\mathrm{Ab}}$ \\
\hline $0,1 \%$ & $4,6 \pm 0,1 \mathrm{Bbc}$ & $6,2 \pm 0,1^{\mathrm{Aa}}$ & $4,6 \pm 0^{\mathrm{Bb}}$ \\
\hline $0 \%$ & $6,4 \pm 0,2^{\mathrm{Aa}}$ & $6,4 \pm 0,2^{\mathrm{Aa}}$ & $6,4 \pm 0,2 \mathrm{Aa}$ \\
\hline \multicolumn{4}{|c|}{24 horas depois } \\
\hline $1 \%$ & $3,6 \pm 0,1^{\mathrm{Bd}}$ & $3,8 \pm 0,1^{\mathrm{Acd}}$ & $3,8 \pm 0^{A c}$ \\
\hline $0,5 \%$ & $3,8 \pm 0,1 \mathrm{Ad}$ & $3,8 \pm 0,2^{\mathrm{Acd}}$ & $3,8 \pm 0^{A c}$ \\
\hline $0,1 \%$ & $3,8 \pm 0,1 \mathrm{Bd}$ & $4,9 \pm 0,1^{\mathrm{Ab}}$ & $3,6 \pm 0,1 \mathrm{Bd}$ \\
\hline $0 \%$ & $4,8 \pm 0,1^{\mathrm{Ab}}$ & $4,8 \pm 0,1^{\mathrm{Ab}}$ & $4,8 \pm 0,1^{\mathrm{Ab}}$ \\
\hline
\end{tabular}

Médias seguidas pela mesma letra maiúscula na linha e mesma letra minúscula na coluna não diferem entre si pelo Teste de Tukey ao nível de $5 \%$ de probabilidade.

O valor de transparência (transmitância a 650 nm) observado por Craig et al. (1989) para pastas de amidos com elevado teor de amilose (como é o caso do amido de ervilha) foi de $5 \%$, enquanto que no presente estudo obteve-se $6,4 \%$.

Após 24 horas da primeira leitura, os valores de transmitância foram menores que no tempo zero devido à retrogradação ocorrida. O comportamento dos três tipos de gomas e das diferentes 
concentrações adicionadas mostrou-se semelhante ao da leitura no tempo zero.

Os valores de transmitância das pastas e géis de amido de grão-de-bico (Tabela 7) foram mais elevados que os de amido de ervilha.

A adição de xantana aumentou a porcentagem de transmitância das pastas de amido de grão-de-bico, independente da concentração utilizada, enquanto que para a goma guar verificouse redução dessa porcentagem. No caso da adição de CMC houve aumento de transmitância nas concentrações $0,1 \%$ e $0,5 \%$ e redução na concentração de $1 \%$.

Os valores de transmitância observados após 24 horas foram menores que no tempo zero. A adição de CMC e de guar a 1\% resultou em menores valores de transmitância das pastas. A dissociação das moléculas de amido durante a gelatinização diminui a habilidade dos grânulos em refletir e, assim, a porcentagem de transmitância da pasta de amido aumenta (SARMENTO, 1997). TABELA 7 - TRANSMITÂNCIA (T\% a 650 nm) DAS PASTAS (0 h) E GÉIS (24 h) DE
AMIDO DE GRÃO-DE-BICO

\begin{tabular}{cccc}
\hline \% de goma & Xantana & Guar & CMC \\
\hline \multicolumn{3}{c}{0 hora } \\
\hline $1 \%$ & $12,9 \pm 0,2^{\mathrm{Aa}}$ & $11,1 \pm 0,4^{\mathrm{Bcd}}$ & $10,5 \pm 0,2^{\mathrm{Cd}}$ \\
$0,5 \%$ & $13,0 \pm 0,2^{\mathrm{Aa}}$ & $11,5 \pm 0,2^{\mathrm{Bbc}}$ & $13,0 \pm 0,2^{\mathrm{Aa}}$ \\
$0,1 \%$ & $13,0 \pm 0,3^{\mathrm{Aa}}$ & $12,1 \pm 0,3^{\mathrm{Bab}}$ & $12,5 \pm 0,1^{\mathrm{ABab}}$ \\
$0 \%$ & $12,3 \pm 0,1^{\mathrm{Ab}}$ & $12,3 \pm 0,1^{\mathrm{Aa}}$ & $12,3 \pm 0,1^{\mathrm{Ab}}$ \\
\hline $1 \%$ & $2^{2} 4$ horas depois & $9,0 \pm 0,2^{\mathrm{Be}}$ \\
\hline $0,5 \%$ & $11,3 \pm 0,3^{\mathrm{Ac}}$ & $9,6 \pm 0,4^{\mathrm{Bf}}$ & $11,4 \pm 0,4^{\mathrm{Ac}}$ \\
$0,1 \%$ & $11,5 \pm 0,1^{\mathrm{Ac}}$ & $10,4 \pm 0,3^{\mathrm{Bde}}$ & $11,2 \pm 0,2^{\mathrm{Ac}}$ \\
$0 \%$ & $11,5 \pm 0,2^{\mathrm{Ac}}$ & $10,4 \pm 0,2^{\mathrm{Bde}}$ & $10,4 \pm 0,3^{\mathrm{Ad}}$ \\
\hline
\end{tabular}

Médias seguidas pela mesma letra maiúscula na linha e mesma letra minúscula na coluna não diferem entre si pelo Teste de Tukey ao nível de $5 \%$ de probabilidade.

Os valores de transmitância das pastas do amido de ervilha foram muito menores que os das pastas do amido de grão-de-bico. Sendo assim, o amido de ervilha apresentou pasta muito mais opaca. Os elevados teores de amilose no amido, segundo Alves, Grossmann e Silva (1999) e Mali et al. (2002) favorecem a retrogradação e resultam em pastas mais opacas.

Grânulos de amidos com teores mais elevados de amilose apresentam expansão e dispersão mais restrita e menor transmitância da pasta quando comparados com os de menor teor de amilose (WANG, WHITE e POLLAK, 1993). O comportamento citado por esses autores foi confirmado no presente trabalho ao se comparar as fontes de amido de ervilha e de grão-de-bico.

\section{CONCLUSÃO}

A adição das gomas afetou a viscosidade da pasta do amido de ervilha, elevando-as de forma proporcional ao aumento da concentração das gomas. Do mesmo modo que a viscosidade, a 
retrogradação do amido também foi aumentada pala adição de gomas. Todas as gomas estudadas provocaram aumento da sinérese e redução da transparência das pastas e géis de amido. Esse fenômeno decorre do elevado teor de amilose do amido de ervilha e da tendência de interações intermoleculares das gomas.

A adição das diferentes gomas afetou de modo não uniforme as propriedades de pasta do amido de grão-de-bico. Houve redução de viscosidade da pasta com a adição de xantana e de CMC, porém aumento com a adição de guar. Todas as gomas, entretanto, reduziram a tendência à retrogradação das pastas. A diminuição mais significativa da sinérese ocorreu pela adição de xantana, devido à redução mais acentuada da tendência à retrogradação da pasta por esse hidrocoloide. $\mathrm{A}$ transparência do amido de grão-de-bico tendeu a se elevar pela adição de xantana e a se reduzir pela adição de guar.

A combinação amido-goma pode ser utilizada para alterar as propriedades funcionais dos amidos. Esse comportamento, entretanto, não foi uniforme para os amidos e nem para os hidrocoloides estudados.

\section{ABSTRACT}

\section{FUNCTIONAL PROPERTIES OF LEGUME STARCH IN COMBINATION WITH HYDROCOLLOIDS}

The objective of this study was to evaluate the effect of addition of hydrocolloids (guar gum, xanthan gum, carboxymethylcellulose) on the physico-chemical and functional characteristics of pea and chickpea starches. The gums were added to the starch at levels of $0,0.1,0.5$ and $1 \%$ of starch weight. The addition of hydrocolloids increased the paste viscosity of pea starch, which is usually very low, and the setback viscosity proportionally with the increase in concentration of gums. The addition of hydrocolloids resulted in reduced transparency of pea starch paste. The addition effect of different hydrocolloids on the pasting properties of chickpea starch was not uniform as for pea starch. However, all the added hydrocolloids reduced the paste setback. The transparency of chickpea starch paste tended to be intensified by adding xanthan and to reduce the addition of guar gum. The association starch-hydrocolloid can be used to change functional properties of starches, increasing the possibility of their use. However, the behavior is not uniform for different starch sources or for different hydrocolloids.

KEY-WORDS: STARCH; CHICKPEA; PEA; HYDROCOLLOIDS; RHEOLOGICAL PROPERTIES.

\section{REFERÊNCIAS}

1 ALVES, R.M.L.; GROSSMANN, M.V.E.; SILVA, R.S.S.F. Gelling properties of extruded yam (Discorea alata) starch. Food Chemistry, v. 67, p. 123-127, 1999.

2 AACC. American Association of Cereal Chemists. Approved methods of the American Association of Cereal Chemists. $9^{\text {th }}$ ed. Saint Paul, 1995

3 AOAC. Association of Official Analytical Chemistry. Official methods of analysis of the AOAC International. $18^{\text {th }}$ ed. Arlington, 2006. $1141 \mathrm{p}$.

4 CEREDA, M.P.; FRANCO, C.M.L.; DAIUTO, E.R.; DEMIATE, I.M.; CARVALHO, L.J.C.B.; LEONEL, M.; VILPOUX, O.F.; SARMENTO, S.B.S. Propriedades gerais do amido. Campinas: Fundação Cargill, 2001. 224 p. (Série Culturas de Tuberosas Amiláceas Latino Americanas, 1).

5 CHAISAWANG, M.; SUPHANTHAKIRA, M. Effects of guar gum and xanthan gum additions on physical and rheological properties of cationic tapioca starch. Carbohydrate Polymers, v. 61, n. 3, p. 288-295, 2005.

6 CHAISAWANG, M.; SUPHANTHAKIRA, M. Pasting and rheological properties of native and anionic tapioca starches as modified by guar gum and xanthan gum. Food Hydrocolloids, v. 20, p. 641-649, 2006.

7 CRAIG, S.A.S.; MANINGAT, C.C.; SEIB, P.A.; HOSENEY, R.C. Starch paste clarity. Cereal Chemistry, v. 66, n. 3, p.173182, 1989.

8 DAVYDOVA, N.I; LEONT'EV, S.P.; GENIN, Y.V; SASO, A.Y; BOGRAHEV, T.Y. Some physico-chemical properties of smooth pea starches. Carbohydrate Polymers, v. 27, p.109-115, 1995.

9 FUNAMI, T.; KATAOKA, Y.; OMOTO, T.; GOTO, Y.; ASAI, I.; NISHINARI, K. Effects of non-ionic polysaccharides on the gelatinization and retrogradation behavior of wheat starch. Food Hydrocolloids, v.19, n. 1, p. 1-13, 2005.

10 HAN, J.Y.; TYLER, R.T. Characterization of pea starches in the presence of alkali and borax. Starch/Stärke, v. 55, n. 6, p. 457-463, 2003. 
11 HOOVER, R.; RATNAYAKE, W.S. Starch characteristics of black bean, chick pea, lentil, navy bean and pinto bean cultivars grown in Canada. Food Chemistry, v. 78, n. 4, p. 489-498, 2002.

12 HOOVER, R.; ZHOU, Y. In vitro and in vivo hydrolysis of legume starch by $\alpha$-amylase and resistant starch formation in legumes - a review. Carbohydrate Polymers, v. 54, n. 4, p. 401-417, 2003.

13 HUANG, J.; SCHOLS, H.A.; SOEST, J.J.G. Van; JIN, Z.; SULMANN, E.; VORAGEN, A.G.J. Physicochemical properties and amylopectin chain profiles of cowpea, chickpea and yellow pea starches. Food Chemistry, v.101, n. 4, p. 1338-1345, 2007.

14 ISO. International Organization for Standardization. Norme internationale: riz détermination de la teneur en amylose. Genève, 1987. 5 p. (ISO 6647)

15 KLUCINEC, J.D.; THOMPSON, D.B. Amylose and amylopectin interact in retrogradation of dispersed high-amylose starches. Cereal Chemistry, v. 76, n. 2, p. 282-291, 1999.

16 KORUS, J.; JUSZCZAK, L.; WITCZAK, M.; ACHEREMOWICZ, B. Influence of selected hydrocolloids on triticale starch rheological properties. International Journal of Food Science and Technology, v. 39, p. 641-652, 2004.

17 LEE, M.H; BAEK, M.H.; CHA, D. S.; PARK, H. J.; LIM, S. T. Freeze-thaw stabilization of sweet potato starch gel by polysaccharide gums. Food Hydrocolloids, v. 16, n. 4, p. 345-352, 2002.

18 MALI, S.; GROSSMANN, M.V.E.; GARCIA, M. A.; MARTINO, M.N.; ZARITZKY, N.E. Microstructural characterization of yam starch films. Carbohydrate Polymers, v. 50, n. 4, p. 379-386, 2002.

19 MUNHOZ, M.P.; WEBER, F.H.; CHANG, Y.K. Influência de hidrocoloides na textura de gel de amido de milho. Ciência e Tecnologia Alimentos, v. 24, n.3, p. 403-406, 2004

20 NEWPORT SCIENTIFIC. Applications manual for the Rapid Visco Analyser. Warriewood: Instrument Support Group, 1998. $92 \mathrm{p}$.

21 PARKER, R.; RING, S.G. Aspects of the physical chemistry of starch. Journal of Cereal Science, v. 34, n. 1, p. 1-17, 2001.

22 POLESI, L. F. Amido resistente obtido a partir de amido de leguminosas e de seus hidrolisados. 2009.128 p. Dissertação (Mestrado em Ciência e Tecnologia de Alimentos) - Escola Superior de Agricultura Luiz de Queiroz, Universidade de São Paulo, Piracicaba, 1997.

23 POLESI, L.F.; SARMENTO, S.B.S.; ANJOS, C.B.P. Composition and characterization of pea and chickpea starches. Brazilian Journal of Food Technology, 2011. No prelo.

24 PONGSAWATMANIT, R.; SRIJUNTHONGSIRI, S. Influence of xanthan gum on rheological properties and freeze-thaw stability of tapioca starch. Journal of Food Engineering, v. 88, p. 137-143, 2008.

25 RATNAYAKE, W.S.; HOOVER, R.; WARKENTIN, T. Pea starch: composition, structure and properties - a review. Starch/ Stärke, v. 54, n. 6, p. 217-234, 2002.

26 RATNAYAKE, W.S.; HOOVER, R.; SHAHIDI, F.; PERERA, C.; JANE, J. Composition, molecular structure, and physicochemical properties of starches from four field pea (Pisum sativum L.) cultivars. Food Chemistry, v. 74, n. 2, p.189-202, 2001

27 SARMENTO, S.B.S. Caracterização da fécula de mandioca (Manihot esculenta Crantz) no período de colheita de cultivares de uso industrial. 1997. 163 p. Tese (Doutorado em Ciências dos Alimentos) - Universidade de São Paulo, São Paulo, 1997.

28 SINGH, N.; SANDHU, K.S.; KAUR, M. Characterization of starches separated from Indian chickpea (Cicer arietinum L.) cultivars. Journal of Food Engineering, v. 63, n. 4, p. 441- 449, 2004.

29 SONG, J.-Y.; KWON, J.-Y.; CHOI, J.; KIM, Y.-C.; SHIN, M. Pasting properties of non-waxy rice starch-hydrocolloid mixtures. Starch/Stärke, v. 58, p. 223-230, 2006.

30 SAS. Statistical Analyses System. User's guide. Version 9.1.3 - Windows Version. Cary, 2002/2003. CD-ROM.

31 SUDHAKAR V.; SINGHAL R.S.; KULKARNI, P.R. Starch-gum interactions: formulations and functionality using amaranth/ corn starch and CMC. Starch/Starke, v. 44, n.10, p. 369-374, 1992.

32 WANG, J.; ZHENGYU, J.; XIAOPING, Y. Preparation of resistant starch from starch-guar gum extrudates and their properties. Food Chemistry, v.101, n. 1, p.20-25, 2007.

33 WANG, Y.J.; WHITE, P.; POLLAK, L. Physicochemical properties of starches from mutant genotypes of the Oh43 Inbred line. Cereal Chemistry, v. 70, n. 2, p. 199-203, 1993. 
34 WEBER, F.H.; QUEIROZ, F.P.C; CHANG, Y.K. Estabilidade de géis de amido de milho normal, ceroso e com alto teor de amilose adicionados de gomas guar e xantana durante os processos de congelamento e descongelamento. Ciência e Tecnologia de Alimentos, v. 28, n. 2, p. 413-417, 2008.

35 WEBER, F.H.; CLERICI, M.T.P.S.; COLLARES-QUEIROZ, F.P.; CHANG, Y.K. Interaction of guar and xanthan gums with starch in the gels obtained from normal, waxy and high-amylose corn starches. Starch/Stärke, v. 61, p. 28-34, 2009. 